\title{
High average brightness electron beam production at Cornell University
}

\author{
Jared Maxson*, Ivan Bazarov, and Bruce Dunham ${ }^{\dagger}$ \\ Cornell Laboratory For Accelerator-based Sciences and Education \\ E-mail: jmm586ecornell.edu
}

Cornell University operates a high average brightness electron photoinjector, using a 350kV DC photogun and a $1.3 \mathrm{GHz}, 5-15 \mathrm{MeV}$ booster linac, as a prototype injector for a $5 \mathrm{GeV}$ Energy Recovery Linac based light source. Our most recent achievements will be described, including the production of world record high-average current from a photoinjector, and demonstration of low emittance. A new $500 \mathrm{kV}$ DC photogun is now being commissioned and its status is reported.

XVth International Workshop on Polarized Sources, Targets, and Polarimetry

September 9 - 13, 2013

Charlottesville, Virginia, USA

\footnotetext{
* Speaker.

${ }^{\dagger}$ For the entire Cornell ERL team. For a full listing, see [1].
} 


\section{Introduction}

Cornell University has been engaged in fundamental research in high brightness electron beam production for more than a decade, towards the goal of constructing a $5 \mathrm{GeV}$ energy recover linac (ERL) synchrotron light source [1]. For such light sources, the principle figure of merit is the normalized average transverse rms beam brightness, which can be defined as:

$$
B_{n, r m s, a v}=\frac{\eta I_{a v}}{\varepsilon_{x, r m s} \varepsilon_{y, r m s}}
$$

where $I_{a v}$ is the average beam current, $\varepsilon_{x, r m s}=\frac{1}{m c} \sqrt{\left\langle x^{2}\right\rangle\left\langle p_{x}^{2}\right\rangle-\left\langle x p_{x}\right\rangle^{2}}$ is the normalized emittance, and $\eta$ is a numerical constant standing for variations in the definition of emittance.

Since the brightness of a linac source is set by its source, Cornell University has constructed a testbed energy injector linac prototype to demonstrate the feasibility of an ERL beam quality. Though the ERL injector does not produce polarized beams, many techniques, including those used in the photocathode electrode source and the production of low-emittance, high current beams, have direct impact on the polarized source community. A schematic of the ERL injector prototype is shown in figure 1, where the beam direction is from right to left. The injector is composed of a $350 \mathrm{kV}$ DC photocathode electron source, driven by $1.3 \mathrm{GHz}$ fiber laser producing 10 's of watts of average power at $520 \mathrm{~nm}$. Following the gun are two emittance compensating solenoids, and a normal conducting $1.3 \mathrm{GHz}$ buncher cavity, used for longitudinal beam compression. Next, 5 2-cell superconducting cavities in a single cryostat accelerate the beam from $350 \mathrm{kV}$ to $5-15 \mathrm{MeV}$. Downstream of the cryostat are diagnostic beamlines, including slit pairs with raster magnets for the measurement of the transverse phase space, as well as a normal conducting deflecting cavity (see below) and a spectrometer chicane to measure the longitudinal phase space on various scintillating view screens. A test merger section (B1 in figure 1) is included, with identical phase space diagnostics. The beam is terminated with a high power beam dump, for use with high current (up to $100 \mathrm{~mA}$ ) operations.

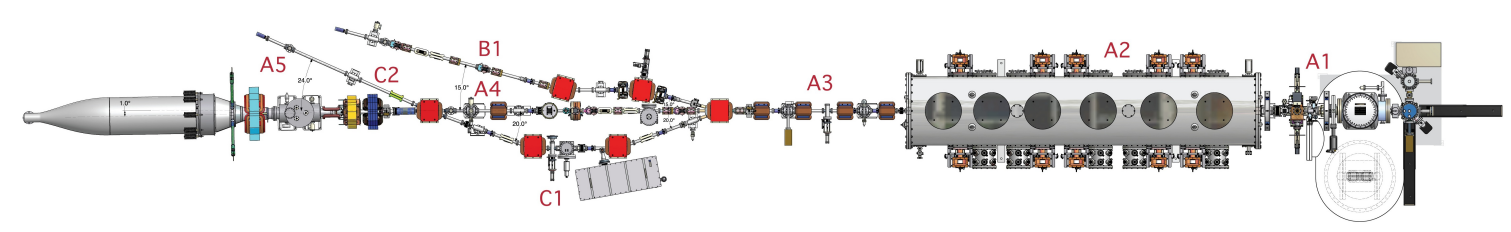

Figure 1: A schematic of the Cornell ERL injector prototype. Beam direction is from right to left. The beamline is a total of $20 \mathrm{~m}$ in length.

The requirements for the Cornell ERL injector as a feasible source for a larger accelerator are given in Table 1. In this proceedings, we will recap the production of high current, and then will describe the demonstration of low emittance. We will conclude with the status of future experiments with a new $500 \mathrm{kV}$ DC gun.

\section{High Current Operations}

One main aim of the Cornell ERL project is the demonstration of $100 \mathrm{~mA}$ of average current. Several milestones, described below, such as the world record current produced from a photoin- 
Table 1: Requirements for the ERL injector Prototype

\begin{tabular}{cccc}
\hline \hline Parameter & Specification & Current Value & Units \\
\hline Average Current & 100 & 75 & $\mathrm{~mA}$ \\
Bunch Charge & 77 & $77(f<1.3 \mathrm{GHz})$ & $\mathrm{pC}$ \\
Energy & 15 & 14 & $\mathrm{MeV}$ \\
Laser Power & $>20(@ 520 \mathrm{~nm})$ & 60 & $\mathrm{~W}$ \\
Gun Voltage & 500 & 350 & $\mathrm{kV}$ \\
RMS norm emit. & $<0.5$ & $0.3($ core $)$ & $\mu \mathrm{m}$ \\
Operational Lifetime & $>1$ & $2.6(@ 65 \mathrm{~mA})$ & days \\
\hline
\end{tabular}

jector, have recently been achieved $[4,6]$. The Cornell injector began its high voltage program by using bulk GaAs photocathodes, activated to negative affinity using $\mathrm{Cs}$ and $\mathrm{NF}_{3}$, as the infrastructure was already in place to grow these cathodes with active areas of various sizes and with an offset from center. The data from the best high current run is plotted in figure 2. Current was increased by smoothly increasing the bunch charge via laser power just above $50 \mathrm{~mA}$, and then the laser power was held constant to measure the decay of the current. The current dips visible in figure 2 are due to SRF input coupler vacuum trips. Furthermore, each trip can be correlated one-to-one with a particular damage spot in the right pane of figure 2, evidence of damage due to the events inside the gun generated from the vacuum burst. The beam energy of this run was $5 \mathrm{MeV}$. The beam loss was estimated to be $I_{\mathrm{loss}} / I_{\mathrm{av}}=2 \times 10^{-8}$ from radiation survey measurements.
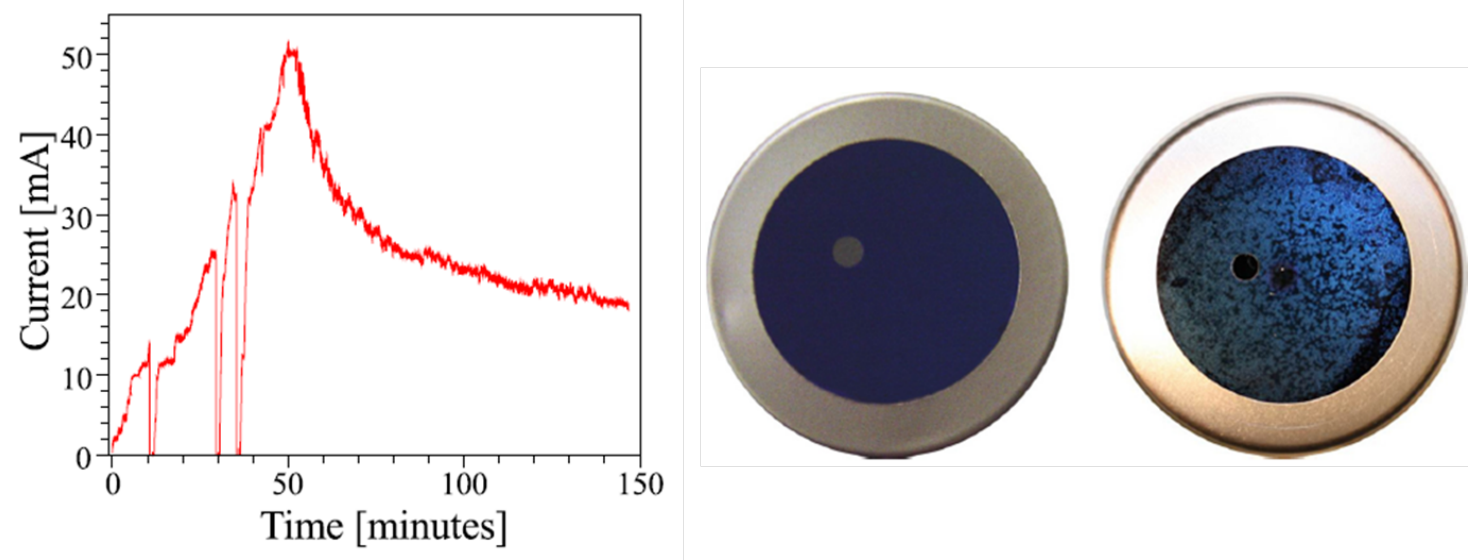

Figure 2: Left: Average beam current, as measured by the gun power supply, as a function of time using the GaAs cathode in the right pane. Right: A photograph of the GaAs photocatode, before and after use. Note small, off center active area. Speckling due to heat cleaning is seen, as well as visible damage from ion back bombardment.

Much more current was delivered with multialkali cathodes, once they could be grown with an off center active area. The Cornell injector has used $\mathrm{CsK}_{3} \mathrm{Sb}, \mathrm{NaKSb}$, and $\mathrm{Cs}_{3} \mathrm{Sb}$ alkali-based photocathodes. The most successful hours-long run was performed with NaKSb. A map of the quantum effficiency $(\mathrm{QE})$ of the cathode prior to the run is shown in figure 3. The current vs. time during the run is shown in figure 3. Current was held at a constant value via fast laser feedback, 
which modulates the laser power to accommodate decreases in QE as well as the fast shift of laser intensity caused by thermal lensing in transport to the gun. The 1/e QE decay measured during this run was $\sim 66$ hours.
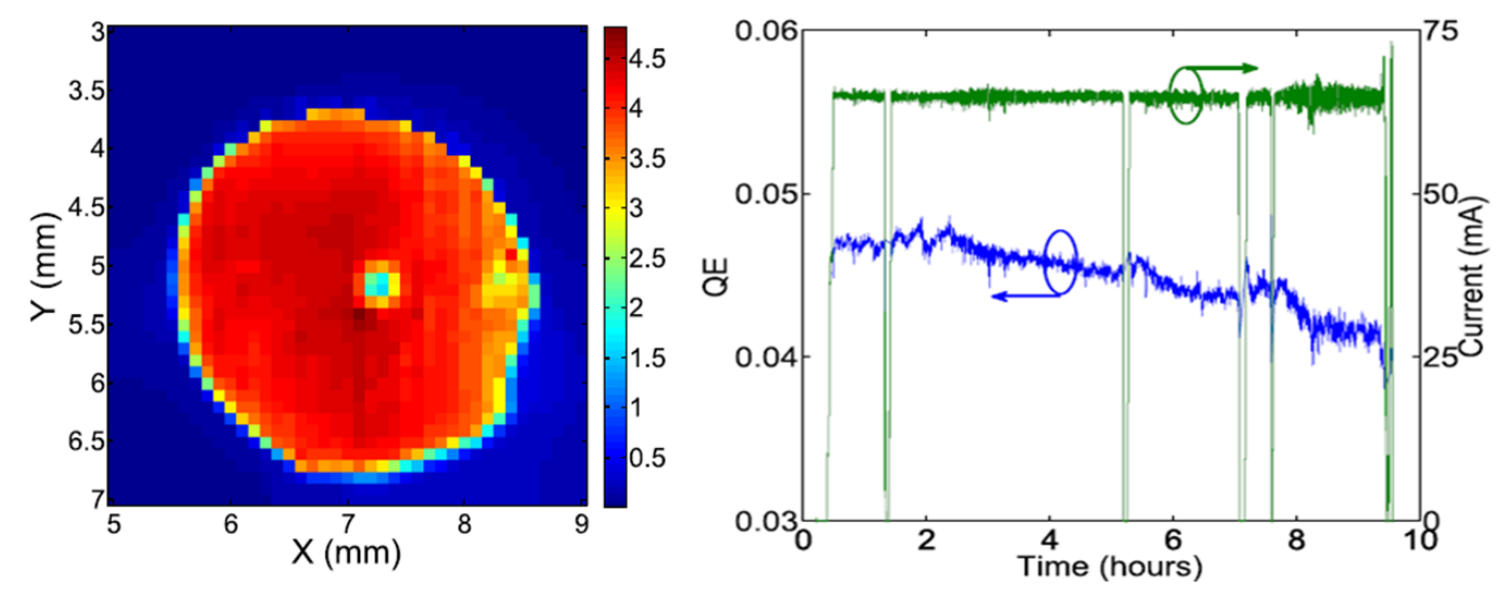

Figure 3: Left: Quantum efficiency map (in percent) of the NaKSb photocathode used in the high current run described above. The low $\mathrm{QE}$ spot was caused by vacuum contamination during loading into the gun. Right: Current and QE vs. time for the run using NaKSb.

\section{Measurement of Low Emittance}

The Cornell ERL photoinjector has also recently demonstrated emittance values below the ERL light source requirement. This work is detailed in [8]. We will summarize the work here. Demonstration of low emittance was an iterative procedure. We first began with an exhaustive model of the beamline using the accelerator simulation code General Particle Tracer [11]. Each acceleration device, including the gun, buncher, and SRF cavities, has a 2 or 3-D field map, time dependent where applicable. In particular, the SRF cavity maps also include the effect of the input couplers, as described in [9]. Each beam optical element is modeled in 2 or 3D, including solenoids, dipoles, quadrupoles, and corrector magnets. The simulation is also given a realistic transverse laser distribution, based on measurements on a screen at a simulated cathode position, as well as temporal laser distribution measured via cross-correlator.

After complete modeling in software, the beam is aligned through each element, up to the secnond SRF cavity, using a low current $(\sim \mu \mathrm{A})$, and bunch charge low enough to neglect space charge effects. The setpoint of each element is oscillated, and the deflection of the beam is measured on a downstream screen. Then, using the transfer matrix formalism of beam optics, the offsets in position and angle are fit to the data taken on the screen. Upstream correctors are adjusted, and the process iterates until offsets in each element are $<10 \mu \mathrm{m}$.

After the cryomodule, the beam is directed into the merger section (B1 in figure 1). The dipole and quadrupole currents are set to provide achromatic transport in the final straight section, where emittance measurements are performed. As mentioned above, emittance measurements are performed with two pairs of $20 \mu \mathrm{m}$ slits (one pair vertical, the other horizontal) and fast scanner magnets that raster the beam in and out of each slit. The transmitted current is read on a Faraday 
cup, and the transmitted current as a function the two correctors currents in the raster can be converted into a projection of the transverse phase space. Furthermore, the transmitted beam passes through a deflection cavity which imparts a vertical momentum as a function of beam arrival time, thus allowing the imaging of the temporal density of the transmitted beamlet on a downstream screen. This allows the measurement of transverse emittance as a function of longitudinal position within the beam.

The beam optics and cavity setpoints are optimized via a multivariate genetic algorithm, as described in [3]. After optimization converges, these settings are loaded into the actual beamline. Then a small scan of the most sensitive optics on the emittance (including the solenoids, buncher voltage and phase, and the first two cavity voltages and phases), until the physical emittance minimum was discovered. The setpoints are then fed back into simulation to check agreement. The results of the emittance optimization and measurement for $77 \mathrm{pC}$ bunch charge are presented in table 2.

Table 2: $90 \%$ and Core normalized RMS emittance for $77 \mathrm{pC}$ charge

\begin{tabular}{cccccccc}
\hline \hline$\varepsilon_{n x, 90}$ & $\varepsilon_{n x, 90}$ & $\varepsilon_{n x, \text { core }}$ & $\varepsilon_{n y, \text { core }}$ & $f_{x, \text { core }}$ & $f_{y, \text { core }}$ & RMS bunch length & RMS energy spread \\
\hline $0.50 \mu \mathrm{m}$ & $0.29 \mu \mathrm{m}$ & $0.28 \mu \mathrm{m}$ & $0.09 \mu \mathrm{m}$ & $64 \%$ & $70 \%$ & $3.0 \mathrm{ps}$ & $0.26 \%$ \\
\hline
\end{tabular}

\section{Future Outlook}

The achievements listed above are more than sufficient proof of concept that the ERL injector can provide a sufficiently bright beam to a next generation ERL light source facility. However, in table 1, perhaps the largest discrepancy between specification and actual value is that of the gun voltage. The question of how large a voltage is required for a particular bunch charge was answered in [2], in which optimizations were performed over a range of bunch charges, for different gun types (DC and SRF) for various gun geometries, voltages, and fields at the photocathode. Optimizations suggest that for bunch charges up to $150 \mathrm{pC}$, the emittance can benefit from $\mathrm{DC}$ voltages up to $\sim 500 \mathrm{kV}$, but beyond, one encounters significant diminishing returns.

For previous designs of DC guns, the ultimate voltage has been limited by ceramic punchthrough, in which field emitted electrons from the high voltage transmitting stalk can puncture the ceramic. The ceramic not only holds off the high voltage, but is a vacuum envelope. Hence, punch-through causes catastrophic failure. Cornell University is currently commissioning a new photoelectron source that has the potential to completely mitigate the problem of punch through [7]. Instead of a single monolithic insulator, the new design features a segmented insulator, for which kovar rings are brazed at the intersection of each joint. See figure 4. Inside vacuum, the kovar rings allow the mounting of copper protection rings, which completely obscure the inner ceramic surface from any field emitted electrons from the stalk. Outside of vacuum, the kovar rings allow the mounting of resistors down the axis of the ceramic, allowing the voltage to be precisely defined at each segment. The resistance in each segment was chose to be $500 \mathrm{M} \Omega$, and there are 14 segments in total. The electrode structure is the standard Pierce type, with a cathode-anode gap of $50 \mathrm{~mm}$, and a cathode focusing angle of 25 degrees. Two $40 \mathrm{~L} / \mathrm{s}$ ion pumps and two 1300L/s chemical getter pumps are mounted the sides of the gun. 
Construction was initially performed following the standards of cleanliness and surface finish established by the SRF community. After mechanical polishing, each high voltage surface has some surface chemistry performed (electropolishing for the 316LN stainless electrodes and a citric acid etch for the copper rings), and is then high pressure rinsed with reverse osmosis purified water for multiple hours. Final assembly takes place in a Class 100 clean room (or better). In an initial build, the gun contained an additional getter pump with window to allow observation of the electrodes during processing. During getter activation, the window cracked, and vacuum was contaminated, causing the sintered getter particles to be released from the pump surface into the gun chamber. As a result of this dust contamination, initial processing was not successful. The gun has been re-cleaned and rebuilt, and at the time of this writing processing is set to begin within weeks. Once the high voltage is reached, we will begin a series of beam experiments to demonstrate a new record low emittance as predicted by detailed beam simulations for the new gun as well as full characterization of the virtual cathode instability limiting a higher charge extraction in photoinjectors $[5,10]$.

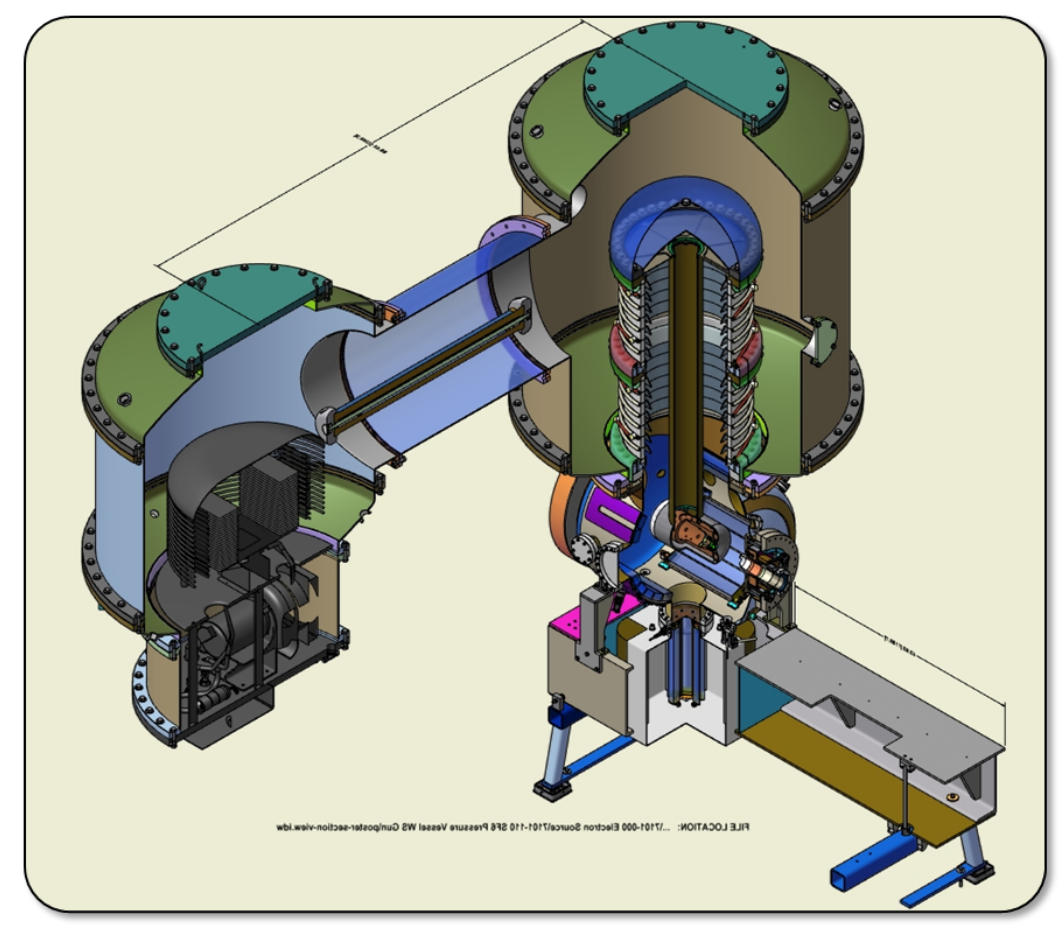

Figure 4: A section view of the new photoelectron source at Cornell University. The outer tanks hold SF6 at $4 \mathrm{~atm}$. The leftmost tank houses a $600 \mathrm{kV}$ Kaiser power supply. Note the segmented insulator design on right, through which a support stalk and pierce electrode structure is held.

\section{Acknowledgments}

This work is supported by the National Science Foundation (Grant No. DMR-0807731 and Gradutate Student Fellowship grant DGE-0707428), as well as the Department of Energy (Grant No. DE-SC0003965). 


\section{References}

[1] G. H. Hoffstaetter et al. Cornell Energy Recovery Linac Project Definition Design Report. 2013. http://www.classe.cornell.edu/ERL/

[2] Ivan V. Bazarov, Allen Kim, Manu N. Lakshmanan, and Jared M. Maxson. Comparison of dc and superconducting rf photoemission guns for high brightness high average current beam production. Phys. Rev. ST Accel. Beams, 14:072001, Jul 2011.

[3] Ivan V. Bazarov and Charles K. Sinclair. Multivariate optimization of a high brightness dc gun photoinjector. Phys. Rev. ST Accel. Beams, 8:034202, Mar 2005.

[4] L. Cultrera, S. Karkare, B. Lillard, A. Bartnik, I. Bazarov, B. Dunham, W. Schaff, and K. Smolenski. Growth and characterization of rugged sodium potassium antimonide photocathodes for high brilliance photoinjector. Applied Physics Letters, 103(10), 2013.

[5] D. H. Dowell, S. Joly, A. Loulergue, J. P. de Brion, and G. Haouat. Observation of space-charge driven beam instabilities in a radio frequency photoinjector. Physics of Plasmas (1994-present), 4(9):3369-3379, 1997.

[6] Bruce Dunham, John Barley, Adam Bartnik, Ivan Bazarov, Luca Cultrera, John Dobbins, Georg Hoffstaetter, Brent Johnson, Roger Kaplan, Siddharth Karkare, Vaclav Kostroun, Yulin Li, Matthias Liepe, Xianghong Liu, Florian Loehl, Jared Maxson, Peter Quigley, John Reilly, David Rice, Daniel Sabol, Eric Smith, Karl Smolenski, Maury Tigner, Vadim Vesherevich, Dwight Widger, and Zhi Zhao. Record high-average current from a high-brightness photoinjector. Applied Physics Letters, 102(3), 2013.

[7] N. Nishimori et al. Generation of a 500-keV electron beam from a high voltage photoemission gun. Applied Physics Letters 102, 234103 (2013).

[8] Colwyn Gulliford, Adam Bartnik, Ivan Bazarov, Luca Cultrera, John Dobbins, Bruce Dunham, Francisco Gonzalez, Siddharth Karkare, Hyeri Lee, Heng Li, Yulin Li, Xianghong Liu, Jared Maxson, Christian Nguyen, Karl Smolenski, and Zhi Zhao. Demonstration of low emittance in the cornell energy recovery linac injector prototype. Phys. Rev. ST Accel. Beams, 16:073401, Jul 2013.

[9] Colwyn Gulliford, Ivan Bazarov, Sergey Belomestnykh, and Valery Shemelin. Asymmetric focusing study from twin input power couplers using realistic rf cavity field maps. Phys. Rev. ST Accel. Beams, 14:032002, Mar 2011.

[10] W. S. Koh, L. K. Ang, and T. J. T. Kwan. Three-dimensional child-langmuir law for uniform hot electron emission. Physics of Plasmas (1994-present), 12(5), 2005.

[11] Pulsar Physics. General particle tracer v. 3.10, 2011. http://www.pulsar.nl/gpt/ 